\title{
Current and Emerging Treatment Strategies for Neuronal Ceroid Lipofuscinoses
}

\author{
Alfried Kohlschütter ${ }^{1}$ (D) Angela Schulz ${ }^{1} \cdot$ Udo Bartsch $^{2} \cdot$ Stephan Storch $^{3}$ (i)
}

Published online: 15 March 2019

(c) The Author(s) 2019

\begin{abstract}
The neuronal ceroid lipofuscinoses comprise a group of neurodegenerative lysosomal storage disorders caused by mutations in at least 13 different genes and primarily affect the brain and the retina of children or young adults. The disorders are characterized by progressive neurological deterioration with dementia, epilepsy, loss of vision, motor disturbances, and early death. While various therapeutic strategies are currently being explored as treatment options for these fatal disorders, there is presently only one clinically approved drug that has been shown to effectively attenuate the progression of a specific form of neuronal ceroid lipofuscinosis, CLN2 disease (cerliponase alfa, a lysosomal enzyme infused into the brain ventricles of patients with CLN2 disease). Therapeutic approaches for the treatment of other forms of neuronal ceroid lipofuscinosis include the administration of immunosuppressive agents to antagonize neuroinflammation associated with neurodegeneration, the use of various small molecules, stem cell therapy, and gene therapy. An important aspect of future work aimed at developing therapies for neuronal ceroid lipofuscinoses is the need for treatments that effectively attenuate neurodegeneration in both the brain and the retina.
\end{abstract}

\section{Introduction}

The neuronal ceroid lipofuscinoses (NCLs) are a heterogeneous group of neurodegenerative lysosomal storage disorders affecting children and young adults. They are characterized by the accumulation of lysosomal storage material and progressive neurological deterioration with dementia, epilepsy, retinopathy, motor disturbances, and early death [1]. While NCLs remain incurable, some NCL forms have recently become amenable to therapies that are reviewed here.

While all NCLs show clinical and neuropathological similarities, each form represents a distinct genetic entity with peculiar pathophysiological characteristics. The present

Udo Bartsch and Stephan Storch shared last authorship.

Alfried Kohlschütter

kohlschuetter@uke.uni-hamburg.de

1 Department of Pediatrics, University Medical Center Eppendorf, Martinistr. 52, 20246 Hamburg, Germany

2 Department of Ophthalmology, Experimental Ophthalmology, University Medical Center Eppendorf, Martinistr. 52, 20246 Hamburg, Germany

3 Department of Pediatrics, Section Biochemistry, University Medical Center Eppendorf, Martinistr. 52, 20246 Hamburg, Germany

\section{Key Points}

The neuronal ceroid lipofuscinoses (NCLs) comprise a group of incurable neurodegenerative storage disorders primarily affecting the brain and the retina of children and young adults, leading to dementia, blindness, epilepsy, and early death.

For one specific form of NCL (CLN2 disease), replacement of the dysfunctional lysosomal enzyme through intraventricular infusion of a functional enzyme (cerliponase alfa) has recently been shown to effectively attenuate the progression of the disease in patients.

Other potential treatment options for NCLs include small molecule therapy, neuroprotection, stem cell therapy, and gene therapy, in addition to enzyme replacement therapy.

As vision loss is among the characteristic clinical symptoms of most NCL variants, treatments are needed that attenuate retinal degeneration in addition to neurodegeneration in the brain. 
Table 1 Neuronal ceroid lipofuscinosis diseases with their age at manifestation, genes, and dysfunctional proteins

\begin{tabular}{|c|c|c|c|c|c|c|c|c|}
\hline \multicolumn{5}{|c|}{ Designation of disease } & \multirow{2}{*}{$\begin{array}{l}\text { OMIM } \\
256730\end{array}$} & \multirow{2}{*}{$\frac{\text { Gene }}{C L N 1(P P T 1)}$} & \multirow{2}{*}{$\begin{array}{l}\text { Protein } \\
\text { Palmitoyl protein thi- } \\
\text { oesterase } 1 \text { (PPT1) }\end{array}$} & \multirow{2}{*}{$\begin{array}{l}\text { Cellular localization } \\
\begin{array}{c}\text { Soluble lysosomal } \\
\text { protein }\end{array}\end{array}$} \\
\hline CLN1 disease & Infantile & Late infantile & Juvenile & Adult & & & & \\
\hline CLN2 disease & & Late infantile & Juvenile & & 204500 & CLN2 (TPP1) & $\begin{array}{l}\text { Tripeptidyl peptidase } \\
\text { (TPP1) }\end{array}$ & $\begin{array}{l}\text { Soluble lysosomal } \\
\text { protein }\end{array}$ \\
\hline CLN3 disease & & & Juvenile & & 204200 & $C L N 3$ & CLN3 & $\begin{array}{l}\text { Lysosomal membrane } \\
\text { protein }\end{array}$ \\
\hline CLN4 disease ${ }^{\mathrm{a}}$ & & & & Adult & 162350 & CLN4 (DNAJC5) & $\begin{array}{l}\text { Cysteine string protein } \\
\alpha(\operatorname{CSP} \alpha)\end{array}$ & $\begin{array}{l}\text { Cytosolic, associated } \\
\text { with vesicular mem- } \\
\text { branes }\end{array}$ \\
\hline CLN5 disease & & Late infantile & Juvenile & Adult & 256731 & CLN5 & CLN5 & $\begin{array}{l}\text { Soluble lysosomal } \\
\text { protein }\end{array}$ \\
\hline CLN6 disease & & Late infantile & & Adult & 601780 & CLN6 & CLN6 & ER membrane protein \\
\hline CLN7 disease & & Late infantile & Juvenile & & 610951 & CLN7 (MFSD8) & CLN7 & $\begin{array}{l}\text { Lysosomal membrane } \\
\text { protein }\end{array}$ \\
\hline CLN8 disease & & Late infantile & Juvenile & & 600143 & CLN8 & CLN8 & ER membrane protein \\
\hline CLN10 disease & Congenital & & Juvenile & Adult & 610127 & $C L N 10(C T S D)$ & Cathepsin D (CTSD) & $\begin{array}{l}\text { Soluble lysosomal } \\
\text { protein }\end{array}$ \\
\hline CLN11 disease & & & & Adult & 614706 & $C L N 11(G R N)$ & Progranulin & $\begin{array}{l}\text { Soluble lysosomal } \\
\text { protein }\end{array}$ \\
\hline CLN12 disease & & & Juvenile & & 610513 & CLN12 (ATP13A2) & ATP 13A2 & $\begin{array}{l}\text { Lysosomal membrane } \\
\text { protein }\end{array}$ \\
\hline CLN13 disease & & & & Adult & 615362 & CLN13 (CTSF) & Cathepsin F & $\begin{array}{l}\text { Soluble lysosomal } \\
\text { protein }\end{array}$ \\
\hline CLN14 disease & Infantile & Late infantile & & & 611725 & CLN14 (KCTD7) & $\begin{array}{l}\text { Potassium chan- } \\
\text { nel tetramerization } \\
\text { domain-containing } \\
\text { protein type } 7\end{array}$ & $\begin{array}{l}\text { Cytosolic, partially } \\
\text { associated with mem- } \\
\text { branes }\end{array}$ \\
\hline
\end{tabular}

$E R$ endoplasmic reticulum

${ }^{a}$ Autosomal dominant inheritance

classification of NCLs is based on the mutated gene (numbered from 1 to 14) and the age at clinical manifestation (Table 1) [2]. With one exception, all known NCLs are transmitted autosomal recessively.

\subsection{Different Neuronal Ceroid Lipofuscinoses (NCL) Diseases}

The different NCL forms and their major pathophysiological and clinical characteristics are summarized below. The diseases are arranged in groups according to the age at which symptoms usually appear. The main alerting symptoms are a newly observed psychomotor abnormality followed by evident dementia in variable combinations with vision loss, epilepsy, and motor deterioration. In rare cases, the clinical presentation is more variable than indicated in this classification; for more details, see the NCL Mutation and Patient Database [3].

\subsubsection{NCL with Onset in the First Year of Life}

Congenital CLN10 disease [4] is associated with dysfunction of the lysosomal enzyme cathepsin D. Patients are born with microcephaly and seizures. The more frequent infantile CLN1 disease [5] is caused by mutations in CLN1 and is associated with dysfunction of the lysosomal enzyme palmitoyl protein thioesterase 1 (PPT1). Onset is in the second half of the first year of life, typically characterized by a decreased muscle tone and decreased social interactions, followed by a dramatic loss of psychomotor functions, myoclonus, seizures, and visual failure. Ultimately, patients develop spasticity and a vegetative state. In rare cases, mutations in CLN14 also cause NCL with infantile onset [6].

\subsubsection{NCL with Late Infantile Onset (Age 2-5 Years)}

The most prevalent NCL form in this group is CLN2 disease ("classic late infantile NCL"), which is caused by mutations in the CLN2 gene, resulting in dysfunction of the lysosomal 
enzyme tripeptidyl peptidase 1 (TPP1). Acquisition of speech may be delayed. First symptoms occur between 2 and 4 years of age and include motor decline with clumsiness and ataxia, deterioration of speech and/or epilepsy. Non-epileptic myoclonus may coexist. After the third year of life, loss of motor function, language, vision, and swallowing ability progresses rapidly, leading to death around the middle teenage years $[7,8]$.

Clinical variants of classic late infantile NCL can also be caused by rare mutations in the CLN1, CLN5, CLN6, CLN7, CLN8, and CLN14 genes and manifest themselves somewhat later and with a slower progression than the classical CLN2 form.

\subsubsection{NCL with Juvenile Onset (Age 5-16 Years)}

Juvenile CLN3 disease ("classic juvenile NCL") is one of the most prevalent NCL forms [9]. It is caused by mutations in the $C L N 3$ gene encoding a lysosomal membrane protein of still unknown function. The disease starts between 4 and 7 years of age with insidious onset of visual failure due to a pigmentary retinopathy. After a considerable interval, progressive cognitive decline and abnormal behavior become apparent. Seizures develop at around 10 years of age followed by a movement disorder and speech and swallowing difficulties. Death usually occurs in the third decade. The clinical course of the disease may be variable even in patients carrying identical mutations, suggesting an influence of modifier genes [10].

Rare forms of NCL manifesting themselves in this age period may also be caused by certain mutations in the $C L N 1$, CLN2, CLN5, CLN7, CLN8, CLN10, or CLN12 genes. Mutations in these genes can also cause NCL in younger patients (see above).

\subsubsection{NCL with Onset in Young Adults (Age 16-30 Years)}

Particularly rare forms of NCL become symptomatic in young adulthood, mostly around the 30th year of life (Table 1). Initial manifestations are cognitive decline and depression, followed by ataxia, parkinsonism, and epilepsy, with or without vision loss [11].

\subsection{Diagnosis of an NCL Disease}

An NCL disorder must be suspected in children and young adults who initially developed normally but then present with an unexplained progressive neurological disorder characterized by dementia, retinopathy, epilepsy, and motor deterioration. Initially, the brain may appear normally developed as assessed by magnetic resonance imaging, but cerebral and cerebellar atrophy will become apparent later in the course of the disease.
The diagnostic approach to a specific NCL form strongly depends on the age at manifestation (Table 1), and the definitive diagnosis is increasingly based on molecular genetic testing. Forms of NCL caused by dysfunctions of lysosomal enzymes (i.e., PPT1 in CLN1, TPP1 in CLN2, and cathepsin D in CLN10 disease) can be diagnosed using enzyme activity assays. In a juvenile-onset disease, a blood smear should be examined for lymphocyte vacuoles, a typical feature of CLN3 disease. In special cases, electron microscopic analyses of blood lymphocytes or tissues may be helpful to confirm a storage disorder.

\section{Present Pharmacological Treatments}

As the NCLs are inherited metabolic disorders, any pharmacological approach to therapy will have to be closely related to the underlying metabolic defect. Specific pharmacological treatments will therefore only be useful for a specific genetic NCL form or to groups of disorders sharing certain metabolic pathways. At present, there is only one clinically approved drug that has been shown to be effective for the treatment of one specific NCL form, CLN2 disease.

\subsection{Cerliponase Alfa}

Cerliponase alfa (Brineura ${ }^{\mathrm{TM}}$, BioMarin Pharmaceutical Inc., Novato, CA, USA) is a recombinant human proenzyme of TPP1, the enzyme affected in CLN2 disease. It was developed by BioMarin Pharmaceutical Inc. and has been globally approved in 2017 for use in patients with CLN2 disease [12]. The drug is administered every other week by intracerebroventricular infusions via a reservoir surgically implanted under the scalp. After infusion, cerliponase alfa enters neuronal cells through mannose 6-phosphate receptor-mediated endocytosis and is subsequently targeted to lysosomes. Here, the proenzyme is activated by removal of a prosegment to become the proteolytic form of TPP1, which cleaves tripeptides from proteins. This proteolytic activity reduces the accumulation of the lysosomal storage material that is closely associated with the neurodegenerative disease.

In phase I/II clinical trials, [13] intracerebroventricular administration of cerliponase alfa has been demonstrated to significantly attenuate progression of motor and language decline in children with CLN2 disease (NCT01907087). Out of 24 patients admitted to the study (aged 3-8 years, median 4 years), 23 completed the study. After a dose-escalation period, they received the drug at a dose of $300 \mathrm{mg}$ every other week for at least 96 weeks (mean $115 \pm 15$ weeks). The rate of decline in a CLN2 clinical rating scale score 
for motor and language functions [14] was used as the primary outcome measure for efficacy. The unadjusted mean rate of decline in this motor-language score per 48-week period was $0.27 \pm 0.35$ points in the 23 treated patients as compared with $2.12 \pm 0.98$ in untreated historical controls [8]. A phase II study enrolling also young, presymptomatic patients (NCT02678689), a long-term extension of the phase I/II study (NCT02485899; NCT02485899), and an expanded access program (NCT02963350) are ongoing.

Adverse effects related to the treatment were generally mild (pyrexia, vomiting, hypersensitivity reactions, rare device-related infections). Convulsions were frequently observed but may have been the result of the underlying brain disease rather than the treatment. All adverse effects where clinically manageable within a few days and did not lead to a discontinuation of treatment.

\section{Therapeutic Strategies: Preclinical Studies and Clinical Trials}

\subsection{Pharmacological Treatments}

\subsubsection{Enzyme Replacement Therapy}

Enzyme replacement therapies using intrathecal administration of recombinant TPP1 have resulted in remarkable therapeutic effects in mouse and canine models of CLN2 disease [15-17]. Administration of a recombinant human TPP1 proenzyme into the lateral ventricles of 2-month-old $C \ln 2$ knockout (ko) mice led to a widespread distribution of the enzyme and increased TPP1 activities throughout the brain. Elevated enzyme activity resulted in reduced autofluorescence and attenuation of tremor amplitudes and neuropathological alterations when compared with vehicle-treated mice [17]. Furthermore, intrathecal application of human TPP1 into 2.5-month-old Dachshunds carrying a null mutation in the $T P P 1$ gene led to a prolonged life span, delayed onset, and progression of neurological symptoms, and reduced brain atrophy in a dose-dependent manner [16]. In a mouse model of CLN1 disease, intrathecal administration of recombinant PPT1 proenzyme to the lumbar spinal cord decreased accumulation of storage material, delayed motor deterioration, and extended the life span in a dose-dependent manner [18]. Intravenous injections of recombinant PPT1 into newborn Pptl ko mice also resulted in an increased life span, and in attenuation of neurodegeneration in the thalamus and a reduction in storage material in visceral tissues of treated mice [19, 20]. Together, results suggest that enzyme replacement therapy represents a promising option for the treatment of patients with CLN1 disease.

\subsubsection{Immunomodulatory Agents}

Neuroinflammation is a hallmark of many NCLs. The efficacy of the antibiotic minocycline to suppress neuroinflammation and neurodegeneration has been analyzed in a naturally occurring CLN6 sheep model [21]. Oral administration of minocycline to pre-symptomatic lambs did not alter neuroinflammation and neurodegeneration. Autoantibodies against several brain antigens including glutamic acid decarboxylase have been detected in sera of $C \ln 3^{\Delta e x}{ }^{-6}$ mice, an animal model of CLN3 disease, and in patients with CLN3 disease [22]. In $C \ln 3^{\Delta e x ~ 1-6}$ mice, immune suppression with the immunosuppressive agent mycophenolate mofetil resulted in reduced levels of serum autoantibodies, attenuation of neuroinflammation, and improvement of motor functions [23]. However, shortterm administration of mycophenolate mofetil to patients with CLN3 disease did not show a clinical benefit [24]. A potential therapeutic benefit of the immunomodulatory compounds fingolimod and teriflunomide was demonstrated in mouse models of CLN1 and CLN3 disease [25]. Oral administration of fingolimod and teriflunomide reduced microgliosis, neuron loss, and brain atrophy in Ppt 1 ko and $C \ln 3$ ko mice. Similarly, intraperitoneal injections of the anti-inflammatory small molecule MW151 into Pptl ko mice led to a decrease in the incidence of seizures [26]. Interestingly, co-administration of MW151 and an adeno-associated virus (AAV) vector encoding human PPT1 improved the therapeutic outcome. The combinatorial treatment resulted in a more pronounced attenuation of neuroinflammation and brain atrophy, and an extended life span when compared with the treatment with the AAVPPT1 vector alone [26].

\subsubsection{Gemfibrozil, Fenofibrate, All-trans-Retinoic Acid, Bezafibrate}

A promising target for therapeutic interventions is the transcription factor EB (TFEB) [27]. Activation of TFEB by increasing its expression and/or nuclear translocation aims to upregulate lysosomal biogenesis and function. Gemfibrozil and fenofibrate, US Food and Drug Administration-approved lipid-lowering drugs, and all-trans-retinoic acid were tested for their potential efficacy to treat CLN2 disease. Gemfibrozil and fenofibrate increased TPP1 messenger RNA and protein levels in normal healthy human and mouse neuronal cells in vitro and in wild-type mouse brains in vivo, but not in neural progenitor cells derived from patients with CLN2 disease $[19,28]$. Similar results were obtained with bezafibrate in lymphoblasts from patients with CLN3 disease [29]. However, gemfibrozil in combination with all-trans-retinoic 
acid increased TFEB expression in cultured fibroblasts from patients with CLN2 disease [30]. Interestingly, application of gemfibrozil to $C \ln 2$ ko mice led to a reduction in storage material in the brain, improved locomotor activities, and a prolonged lifespan [30]. Of note, the combination of gemfibrozil and all-trans-retinoic acid (PLX-100, Polaryx Therapeutics, Paramus, NJ, USA) has recently received an orphan drug designation by the Food and Drug Administration for the treatment of NCLs.

\subsubsection{Trehalose, MK2206}

The disaccharide trehalose and the drug MK2206 inhibit the serine/threonine kinase Akt independently of the mechanistic target of rapamycin complex 1 , resulting in increased nuclear translocation of TFEB [31]. Oral trehalose treatment of $C \ln 3^{\Delta e x 7 / 8}$ mice led to clearance of lysosomal storage, reduced neuroinflammation and neurodegeneration, and a prolonged life span [31]. Along the same line, intraperitoneal application of the Akt inhibitor MK2206 to $C \ln 3{ }^{\Delta e x 7 / 8}$ mice resulted in nuclear translocation of TFEB and upregulation of lysosomal and autophagy genes in the brain [31]. Furthermore, MK2206 reduced storage material in fibroblasts from patients with CLN1, CLN2, CLN3, and CLN7 disease, suggesting a potential therapeutic effect of Akt inhibition in multiple NCLs.

\subsubsection{Cysteamine, $\mathrm{N}$-Acetylcysteine, $\mathrm{N}$-(tert-Butyl) Hydroxylamine}

In CLN1 disease, PPT1 dysfunction leads to neuronal death and intracellular accumulation of granular osmiophilic deposits [32]. Loss of PPT1 impairs the cleavage of the thioester linkage in palmitoylated proteins, and as a consequence their subsequent degradation by lysosomal hydrolases. The thioester linkage is susceptible to nucleophilic attack by substances such as phosphocysteamine and $N$-acetylcysteine. Phosphocysteamine has been shown to decrease granular osmiophilic deposit formation in cultured lymphoblasts and fibroblasts from patients with CLN1 disease [33]. Furthermore, treatment of Pptl ko mice with phosphocysteamine improved the outcome of a central nervous system (CNS)-directed gene therapy, albeit to only a limited extent [34]. Similarly, oral administration of cysteamine bitartrate and $N$-acetylcysteine to patients with CLN1 disease had only a limited therapeutic impact [35].

The small molecule $N$-(tert-Butyl) hydroxylamine (NtBuHA) mimics the function of thioesterases including PPT1. In Ppt1 ko mice, NtBuHA has been shown to reduce lysosomal storage in the brain, to improve motor functions, and to moderately extend the life span of treated animals [36]. Furthermore, NtBuHA has been demonstrated to reduce the amount of palmitoylated proteins and storage material in cultured cells from patients with CLN1 disease [36].

\subsubsection{Phosphodiesterase-4 Inhibitors}

Based on the observation that cyclic adenosine monophosphate levels are significantly reduced in various brain regions of $C \ln 3^{\Delta e x 7 / 8}$ mutant mice, a recent study has evaluated the use of the phosphodiesterase 4 inhibitors rolipram, roflumilast, and PF-06266047 for the treatment of CLN3 disease [37]. In $C \ln 3^{\Delta e x 7 / 8}$ mice that had received daily subcutaneous applications of rolipram or oral applications of roflumilast or PF-06266047, levels of the lysosomal-associated membrane protein 1 were reduced, astrogliosis and microgliosis were attenuated, and motor functions were improved, suggesting that the administration of phosphodiesterase 4 inhibitors might represent a novel therapeutic strategy for the treatment of patients with CLN3 disease.

\subsection{7 a-Amino-3-Hydroxy-5-Methyl-4-Isoxazolepropionic Acid/N-Methyl-D-Aspartate-Type Glutamate Receptor Antagonists}

Studies on $C \ln 3^{\Delta e x I-6}$ mice have revealed a dysregulation of the $\alpha$-amino-3-hydroxy-5-methyl-4-isoxazolepropionic acid-type glutamate receptor activity in cerebellar granule cells [38]. Intraperitoneal injections of the non-competitive $\alpha$-amino-3-hydroxy-5-methyl-4-isoxazolepropionic acid receptor antagonist, EGIS-8332, led to a short-term improvement in motor coordination, but had no beneficial impact on neuroinflammation and neurodegeneration in the CLN3 mouse model [39]. Similarly, inhibition of $N$-methylD-aspartate-type glutamate receptors by memantine resulted in only a short-term improvement of motor skills [40].

\subsection{Stem Cell Therapy}

Stem cell therapy for the treatment of NCLs caused by dysfunctional lysosomal enzymes is based on the rationale that grafted human neural stem cells from a healthy donor will differentiate into neurons and glia in the recipient patient's brain where they express and secrete functional lysosomal enzymes, which can be internalized via mannose 6-phosphate receptors by neighboring mutant neural cells. Transplantation of genetically non-modified human CNS stem cells into the brain of a CLN1 mouse model resulted in the expression of PPT1 in the brain, a reduction in autofluorescent storage material, reduced loss of hippocampal and cortical neurons, and improved locomotor activities when compared with non-treated mice [41]. Based on these data, a 
phase I clinical trial (NCT00337636) was performed on two patients with CLN1 disease and four patients with CLN2 disease at advanced stages using purified, allogeneic, fetal, human CNS stem cells. The human CNS stem cells were transplanted into the cerebral hemispheres and lateral ventricles [42]. While there were no adverse effects associated with the surgery, immunosuppression, and cell transplantation, the treatment did not slow disease progression as judged from a comparison of treated patients with natural history controls.

\subsection{Gene Therapy}

\subsubsection{Gene Therapy in Preclinical Studies}

Gene therapy approaches have shown promising results in animal models of various NCLs. For instance, newborn Pptl ko mice that were treated with multiple intracranial injections of an AAV2 vector encoding human PPT1 exhibited PPT1 enzyme activity near the injection sites, reduction in storage material, attenuation of neurodegeneration, and improvement in various behavioral tests when compared with control mutant mice. A decrease in the incidence of seizures or an increase in longevity were, however, not observed in the treated mice [43]. Multiple intracranial injections of an AAV5-PPT1 vector into young Pptl ko mice also resulted in attenuation of neurodegeneration and improvement of locomotor performance, and additionally extended the life span of treated animals [44]. Therapeutic effects on motor performance and longevity were significantly more pronounced when the gene therapy approach was combined with bone marrow cell transplantations, while bone marrow cell transplantations alone showed no therapeutic effects [44]. Furthermore, injections of AAV9-PPT1 into the brain or spinal cord of Pptl ko mice resulted in attenuation of neuropathological alteration in the respective CNS regions, and in improved motor function and a prolonged life span. Interestingly, simultaneous treatments of the brain and the spinal cord resulted in a significantly better therapeutic outcome than treatments of either CNS region alone, indicating the need for targeting the spinal cord in addition to the brain to develop effective therapies for NCLs [45].

Intraventricular injections of an AAV2-TPP1 vector into a canine model of CLN2 disease resulted in high expression levels of TPP1 in ependymal cells and a widespread distribution of the enzyme in the brain [46]. Treated dogs showed reduced accumulation of storage material, attenuated astrogliosis, delayed onset and progression of neurological symptoms, and a prolonged life span. The CLN2-related non-neuronal pathology in visceral tissues was not prevented by the AAV2-mediated TPP1 expression in the brain [47]. Finally, a safety and tolerability study was conducted on African green monkeys using intracerebral injections of
AAVrh10-TPP1 [48]. Experiments revealed long-term expression of TPP1 in the brain with a 1.6-fold higher TPP1 activity than in phosphate buffered saline-injected controls, and no adverse effects related to the treatment except moderate white matter edema and inflammation near the injection sites of the vector.

The impact of a gene therapy approach for the treatment of CLN3 disease was studied in $C \ln 3^{\Delta e x 7 / 8}$ mice that had received intravenous injections of an AAV9-CLN3 vector [49]. Analysis of treated mice revealed a reduction in lysosomal storage material in the brain, reduced astrogliosis and microgliosis, and improved motor function. In another study, injections of an AAVrh.10-CLN3 vector into the brain parenchyma of newborn $C \ln 3^{\Delta e x 7 / 8}$ mice also resulted in a partial correction of the neurological phenotype as shown by the reduction in storage material and attenuation of astrogliosis [50].

The therapeutic potential of a gene therapy approach for the treatment of CLN5 disease was analyzed in a naturally occurring sheep model of this condition using lentiviral or AAV9 vectors encoding ovine $C L N 5$. When viral vectors were administered to pre-symptomatic animals, brain atrophy and visual decline were significantly delayed, and life span dramatically prolonged. Of note, attenuation of disease progression was also observed when the treatment of animals was started at a symptomatic stage [51].

The impact of an AAV vector-mediated expression of cathepsin D (CtsD) in either the brain or visceral organs or both on disease progression was studied in a $C t s d$ ko mouse [52]. Treatment of the CNS led to a widespread distribution of CtsD in neurons remote from the injection site, indicating Ctsd secretion from transduced cells and re-uptake of the enzyme by distant cells. Expression of CtsD in the brain prevented accumulation of ceroid lipopigments and activation of microglia, and significantly extended the life span of mutant mice. Interestingly, treatment of the brain additionally delayed progression of the visceral pathology, indicating that the enzyme is drained from the brain to the periphery. In contrast, expression of CtsD in peripheral organs had no effect on visceral pathology and life span. Finally, a recent study has demonstrated that an AAV-mediated delivery of progranulin (Grn) to the cerebral hemispheres of Grn ko mice, an animal model of CLN11 disease, reduced the amount of ceroid lipopigments, attenuated microgliosis, improved lysosomal function, and corrected abnormal CtsD activity [53].

\subsubsection{Gene Therapy in Clinical Trials}

A gene therapy clinical trial (NCT00151216) using intracranial injections of an AAV2-CLN2 vector for the treatment of CLN2 disease has been completed [54]. Neurological rating and quantitative magnetic resonance imaging 
revealed attenuation of neurological impairment and a delay in the decline of magnetic resonance imaging parameters, indicating that the treatment slowed disease progression in patients with CLN2 disease. A phase I/II clinical trial (NCT01161576) using intracranial injections of an AAVrh.10-CLN2 vector started in 2010. The aim of the clinical trial is to evaluate the safety, potential toxicity, and therapeutic efficacy of the treatment. Another clinical trial (NCT01414985) in patients with CLN2 disease using the same protocol and expanded eligibility criteria is ongoing. A phase I/IIa gene therapy clinical trial (NCT02725580) has also been started for the treatment of CLN6 disease. Patients receive intrathecal injections of an AAV9-CLN6 vector, results are pending. Recently, another phase I/IIa gene therapy clinical trial has been started for the treatment of CLN3 disease. Patients receive intrathecal injections of an AAV9CLN3 vector, enrollment is ongoing (NCT03770572).

\section{Treatment Strategies for Retinal Degeneration and Vision Loss}

Currently, there is no effective treatment for retinal degeneration in NCLs. However, preclinical studies have demonstrated the efficacy of various therapeutic strategies to attenuate the progression of retinal degeneration and vision loss in animal models of various NCL variants.

\subsection{Gene Augmentation Therapy}

The $n c l f$ mouse, a naturally occurring animal model of CLN6 disease [55], is characterized by an early-onset loss of photoreceptor cells. A recent study has evaluated the efficacy of a gene augmentation approach to prevent visual deterioration in this mutant, and unexpectedly found that an AAV vector-mediated expression of CLN6 in photoreceptor cells had no effect on retinal degeneration and visual impairment [56]. However, deterioration of retina structure and function was significantly delayed when CLN6 was expressed in bipolar cells, suggesting that bipolar cell dysfunction is the cause of photoreceptor loss in the nclf mutant. Similar studies aimed at evaluating the efficacy of gene augmentation strategies to treat vision loss in the most prevalent NCL variant, CLN3 disease, are hampered by the lack of animal models displaying a pronounced retinal dystrophy. A recent study has therefore used induced pluripotent stem cells from CLN3 patients to generate retinal neurons. Transduction of these patient-specific neurons with an AAV2-CLN3 vector resulted in restoration of full-length CLN3 protein expression. Furthermore, subretinal injections of the vector into wild-type mice resulted in expression of full-length $C L N 3$ transcripts, with no obvious adverse effects on treated retinas [57].

\subsection{Neuroprotection}

The mnd mutant mouse is a naturally occurring animal model of CLN8 disease [58]. Intravitreal transplantations of neurally differentiated murine embryonic stem cells into this mutant resulted in extensive integration of donor cells into the dystrophic host retinas, a significant decrease in the number and size of autofluorescent storage bodies, and a significant increase in the number of surviving photoreceptor cells when compared with sham-injected control animals [59]. The mechanism of how the grafted cells exerted these effects on the mnd retina is unknown. To directly analyze whether the administration of a neuroprotective factor can attenuate retinal degeneration in NCL, Jankowiak et al. [60] generated clonal neural stem cell lines overexpressing the ciliary neurotrophic factor, a cytokine known to effectively promote photoreceptor cell survival [61]. The ciliary neurotrophic factor expressing neural stem cells was intravitreally grafted into a CLN6 mouse model prior to the onset of photoreceptor loss. Analyses of retinas 6 weeks after the cell transplantation revealed a significantly higher number of photoreceptor cells in ciliary neurotrophic factor-treated eyes than in contralateral eyes with grafted control cells [60], indicating that neuroprotective approaches represent another strategy to attenuate retinal degeneration in NCL variants caused by defective transmembrane proteins.

\subsection{Immunomodulatory Therapy}

Emerging evidence suggests that innate and adaptive immune responses are critical determinants of disease progression in NCLs. Reactive microgliosis and immigration of lymphocytes into brain tissue are characteristic features of the neurological phenotype of Ppt1 ko mice [62]. Analyses of Ppt 1 ko mice additionally deficient in RAG-1, and thus devoid of lymphocytes, combined with reconstitution experiments identified CD8-positive T lymphocytes as critical pathogenic mediators of the neurological phenotype. Of interest in the present context, inactivation of lymphocytes resulted in significant attenuation of retinal ganglion cell loss and visual deterioration as assessed in optokinetic tracking experiments [62]. Along the same line, the authors subsequently studied the functional relevance of elevated sialoadhesin levels, a sialic acid binding immunoglobulinlike lectin implicated in immunomodulation and inflammation, on activated microglia/macrophages in Pptl ko and $C \ln 3^{e x ~}{ }^{1-6}$ mice. Again, using a genetic approach, the study showed that sialoadhesin deficiency partially prevented retinal ganglion cell loss in both mouse models, and significantly attenuated thinning of inner retinal layers in Pptl ko mice at the advanced stage of the disease [63]. Based on the combined findings, the authors next evaluated the therapeutic impact of two immunomodulatory compounds, 
fingolimod and teriflunomide (see also Sect. 3.1.2), on the progression of retinal degeneration in Ppt1 and aged $C \ln 3$ ko mice, and again found significant attenuation of retinal thinning and retinal ganglion cell loss [25]. Immune modulatory compounds have also been tested in the nclf mouse [64]. Dietary supplementation with the natural immunomodulators docosahexaenoic acid or curcumin attenuated reactive microgliosis in the mutant retina and partially preserved retinal function as assessed in optokinetic tracking experiments and electroretinogram recordings. Furthermore, docosahexaenoic acid, but not curcumin, mitigated thinning of the photoreceptor cell layer [64].

\subsection{Enzyme Replacement Strategies}

While enzyme replacement strategies that specifically target the brain have been demonstrated to effectively delay disease progression in various animal models of NCL and recently in patients with CLN2 disease (see above), they will unlikely have a significant impact on the onset and progression of retinal pathology. In fact, an AAV vector-mediated expression of CtsD in the brain of CtsD-deficient mice effectively attenuated neurodegeneration in the brain, but not in the retina [52]. Similarly, administration of TPP1 to the brain of a canine CLN2 model markedly ameliorated most neurological symptoms [16, 46] (see also Sect. 3.1.1), but did not prevent retinal degeneration $[65,66]$. To evaluate the therapeutic impact of a sustained intraocular administration of a lysosomal enzyme on retina pathology, Griffey and colleagues used an AAV2 vector to express human PPT1 in the retina of Ppt1 ko mice [67]. Intravitreal injections of the vector resulted in expression of the enzyme mainly in retinal ganglion cells, and in enzyme levels that markedly exceeded those normally found in wild-type eyes. Importantly, the treatment delayed deterioration of retina function as assessed by electroretinogram recordings. Photoreceptor numbers were, however, not significantly different between treated and control Ppt 1 ko retinas [67]. The efficacy of a cell-based enzyme replacement strategy to attenuate retinal degeneration has recently been analyzed in a canine CLN2 model [65]. A single intravitreal injection of autologous mesenchymal stem cells modified to overexpress TPP1 resulted in significant preservation of retina structure and function, suggesting that intraocular transplantations of ex-vivo modified cells represent another promising means to deliver therapeutically relevant quantities of a lysosomal enzyme to dystrophic retinas [65].

\section{Palliative Care}

Palliative therapies are of utmost importance in these chronic diseases and represent a significant challenge owing to the multiplicity of symptoms and affected systems.
Moreover, treatment is difficult as most patients have poor vision and may not be able to communicate verbally. Some aspects of palliative therapy, including drug treatment for epilepsy, myoclonus, and spasticity, are specifically related to the specific genetic diagnosis. A multidisciplinary approach is usually indicated [1, 68, 69]. For juvenile blind patients with CLN3 disease with progressive loss of cognitive and other abilities, who present a great challenge to education, consolidated specific experience has been accumulated [70].

\section{Conclusions}

Neuronal ceroid lipofuscinoses comprise a genetically heterogeneous group of fatal neurodegenerative lysosomal storage disorders characterized by the accumulation of autofluorescent storage material, cognitive deficits, seizures, brain atrophy, vision loss through retinopathy, and premature death. While NCLs are regarded to be incurable, a variety of treatment strategies have been developed in animal models, some of them with a promising therapeutic outcome. An effective treatment option for NCL types caused by dysfunctions of soluble lysosomal enzymes is the replacement of the defective enzyme by infusion of the recombinant protein, virus-mediated gene transfer, or cell-based approaches. A recent report demonstrating the efficacy of an enzyme replacement therapy to slow disease progression in patients with CLN2 disease is in line with this view. The development of effective therapies for NCLs caused by mutations in transmembrane proteins, in comparison, is more challenging. Potential treatment options include gene augmentation strategies, immunomodulatory therapies, neuroprotection, or small-molecule therapies. Results from preclinical studies suggest that a combination of different treatment strategies might be required to achieve significant therapeutic outcomes. Preclinical studies also suggest that therapeutic strategies have to target the spinal cord, the retina, and eventually peripheral organs in addition to the brain to establish effective therapies for NCLs.

\section{Compliance with Ethical Standards}

Funding No sources of funding were received for the preparation of this review. The Open Access fee was paid from scientific overhead costs awarded to Dr. Angela Schulz.

Conflict of interest Alfried Kohlschütter and Angela Schulz have been consultants to BioMarin Pharmaceutical Inc., Novato, CA, USA during a clinical trial of cerliponase A and have received fees, honoraria, and travel support. Stephan Storch and Udo Bartsch have no conflicts of interest that are directly relevant to the content of this review. 
Open Access This article is distributed under the terms of the Creative Commons Attribution-NonCommercial 4.0 International License (http://creativecommons.org/licenses/by-nc/4.0/), which permits any noncommercial use, distribution, and reproduction in any medium, provided you give appropriate credit to the original author(s) and the source, provide a link to the Creative Commons license, and indicate if changes were made.

\section{References}

1. Nita DA, Mole SE, Minassian BA. Neuronal ceroid lipofuscinoses. Epileptic Disord. 2016;18:73-88.

2. Williams RE, Mole SE. New nomenclature and classification scheme for the neuronal ceroid lipofuscinoses. Neurology. 2012;79:183-91.

3. NCL Mutation and Patient Database. 1998-2018. https://www. ucl.ac.uk/ncl/mutation.shtml. Accessed Feb 2019.

4. Cooper JD, Partanen S, Siintola E, Steinfeld R, Strömme P, Tyynelä J. CLN10. In: Mole SE, Williams RE, Goebel HH, editors. The neuronal ceroid lipofuscinoses (Batten disease). 2nd ed. Oxford: Oxford University Press; 2011. p. 203-12.

5. Autti T, Cooper JD, Van Diggelen OP, Haltia M, Jalanko A, Kitzmüller C, et al. CLN1. In: Mole SE, Williams RE, Goebel $\mathrm{HH}$, editors. The neuronal ceroid lipofuscinoses (Batten disease). 2nd ed. Oxford: Oxford University Press; 2011. p. 55-79.

6. Khan A, Chieng KS, Baheerathan A, Hussain N, Gosalakkal J. Novel CLN1 mutation with atypical juvenile neuronal ceroid lipofuscinosis. J Pediatr Neurosci. 2013;8:49-51.

7. Kohlschütter A, Schulz A. CLN2 disease (classic late infantile neuronal ceroid lipofuscinosis). Pediatr Endocrinol Rev. 2016;13:682-8.

8. Nickel M, Simonati A, Jacoby D, Lezius S, Kilian D, Van de Graaf $\mathrm{B}$, et al. Disease characteristics and progression in patients with late-infantile neuronal ceroid lipofuscinosis type 2 (CLN2) disease: an observational cohort study. Lancet Child Adolesc Health. 2018;2:582-90.

9. Åberg L, Autti T, Braulke T, Cooper JD, van Diggelen OP, Jalanko A, et al. CLN3. In: Mole SE, Williams RE, Goebel HH, editors. The neuronal ceroid lipofuscinoses (Batten disease). 2nd ed. Oxford: Oxford University Press; 2011. p. 110-39.

10. Lebrun AH, Moll-Khosrawi P, Pohl S, Makrypidi G, Storch S, Kilian D, et al. Analysis of potential biomarkers and modifier genes affecting the clinical course of CLN3 disease. Mol Med. 2011;17:1253-61.

11. Berkovic SF, Staropoli JF, Carpenter S, Oliver KL, Kmoch S, Anderson GW, et al. Diagnosis and misdiagnosis of adult neuronal ceroid lipofuscinosis (Kufs disease). Neurology. 2016;87:579-84.

12. Markham A. Cerliponase alfa: first global approval. Drugs. 2017;77:1247-9.

13. Schulz A, Ajayi T, Specchio N, de Los Reyes E, Gissen P, Ballon D, et al. Study of intraventricular cerliponase alfa for CLN2 disease. N Engl J Med. 2018;378:1898-907.

14. Wyrwich KW, Schulz A, Nickel M, Slasor P, Ajayi T, Jacoby DR, et al. An adapted clinical measurement tool for the key symptoms of CLN2 disease. JIEMS. 2018;6:1-7.

15. Passini MA, Dodge JC, Bu J, Yang W, Zhao Q, Sondhi D, et al. Intracranial delivery of CLN2 reduces brain pathology in a mouse model of classical late infantile neuronal ceroid lipofuscinosis. J Neurosci. 2006;26:1334-42.

16. Katz ML, Coates JR, Sibigtroth CM, Taylor JD, Carpentier M, Young WM, et al. Enzyme replacement therapy attenuates disease progression in a canine model of late-infantile neuronal ceroid lipofuscinosis (CLN2 disease). J Neurosci Res. 2014;92:1591-8.

17. Chang M, Cooper JD, Sleat DE, Cheng SH, Dodge JC, Passini MA, et al. Intraventricular enzyme replacement improves disease phenotypes in a mouse model of late infantile neuronal ceroid lipofuscinosis. Mol Ther. 2008;16:649-56.

18. Lu JY, Nelvagal HR, Wang L, Birnbaum SG, Cooper JD, Hofmann SL. Intrathecal enzyme replacement therapy improves motor function and survival in a preclinical mouse model of infantile neuronal ceroid lipofuscinosis. Mol Genet Metab. 2015;116:98-105.

19. Ghosh A, Corbett GT, Gonzalez FJ, Pahan K. Gemfibrozil and fenofibrate, Food and Drug Administration-approved lipid-lowering drugs, up-regulate tripeptidyl-peptidase 1 in brain cells via peroxisome proliferator-activated receptor alpha: implications for late infantile Batten disease therapy. J Biol Chem. 2012;287:38922-35.

20. Hu J, Lu JY, Wong AM, Hynan LS, Birnbaum SG, Yilmaz DS, et al. Intravenous high-dose enzyme replacement therapy with recombinant palmitoyl-protein thioesterase reduces visceral lysosomal storage and modestly prolongs survival in a preclinical mouse model of infantile neuronal ceroid lipofuscinosis. Mol Genet Metab. 2012;107:213-21.

21. Kay GW, Palmer DN. Chronic oral administration of minocycline to sheep with ovine CLN6 neuronal ceroid lipofuscinosis maintains pharmacological concentrations in the brain but does not suppress neuroinflammation or disease progression. J Neuroinflamm. 2013;10:97.

22. Chattopadhyay S, Ito M, Cooper JD, Brooks AI, Curran TM, Powers JM, et al. An autoantibody inhibitory to glutamic acid decarboxylase in the neurodegenerative disorder Batten disease. Hum Mol Genet. 2002;11:1421-31.

23. Seehafer SS, Ramirez-Montealegre D, Wong AM, Chan $\mathrm{CH}$, Castaneda J, Horak M, et al. Immunosuppression alters disease severity in juvenile Batten disease mice. J Neuroimmunol. 2010;230:169-72.

24. Augustine EF, Beck CA, Adams HR, Defendorf S, Vierhile A, Timm D, et al. Short-term administration of mycophenolate is well-tolerated in CLN3 disease (juvenile neuronal ceroid lipofuscinosis). JIMD Rep. 2019;43:117-24.

25. Groh J, Berve K, Martini R. Fingolimod and teriflunomide attenuate neurodegeneration in mouse models of neuronal ceroid lipofuscinosis. Mol Ther. 2017;25:1889-99.

26. Macauley SL, Wong AM, Shyng C, Augner DP, Dearborn JT, Pearse Y, et al. An anti-neuroinflammatory that targets dysregulated glia enhances the efficacy of CNS-directed gene therapy in murine infantile neuronal ceroid lipofuscinosis. J Neurosci. 2014;34:13077-82.

27. Ballabio A. The awesome lysosome. EMBO Mol Med. 2016;8:73-6.

28. Lojewski X, Staropoli JF, Biswas-Legrand S, Simas AM, Haliw L, Selig MK, et al. Human iPSC models of neuronal ceroid lipofuscinosis capture distinct effects of TPP1 and CLN3 mutations on the endocytic pathway. Hum Mol Genet. 2014;23:2005-22.

29. Hong M, Song KD, Lee HK, Yi S, Lee YS, Heo TH, et al. Fibrates inhibit the apoptosis of Batten disease lymphoblast cells via autophagy recovery and regulation of mitochondrial membrane potential. Vitro Cell Dev Biol Anim. 2016;52:349-55.

30. Ghosh A, Jana M, Modi K, Gonzalez FJ, Sims KB, Berry-Kravis $\mathrm{E}$, et al. Activation of peroxisome proliferator-activated receptor alpha induces lysosomal biogenesis in brain cells: implications for lysosomal storage disorders. J Biol Chem. 2015;290:10309-24.

31. Palmieri M, Pal R, Nelvagal HR, Lotfi P, Stinnett GR, Seymour ML, et al. mTORC1-independent TFEB activation via Akt inhibition promotes cellular clearance in neurodegenerative storage diseases. Nat Commun. 2017;8:14338. 
32. Mitchison HM, Hofmann SL, Becerra CH, Munroe PB, Lake BD, Crow YJ, et al. Mutations in the palmitoyl-protein thioesterase gene (PPT; CLN1) causing juvenile neuronal ceroid lipofuscinosis with granular osmiophilic deposits. Hum Mol Genet. 1998;7:291-7.

33. Zhang Z, Butler JD, Levin SW, Wisniewski KE, Brooks SS, Mukherjee AB. Lysosomal ceroid depletion by drugs: therapeutic implications for a hereditary neurodegenerative disease of childhood. Nat Med. 2001;7:478-84.

34. Roberts MS, Macauley SL, Wong AM, Yilmas D, Hohm S, Cooper JD, et al. Combination small molecule PPT1 mimetic and CNS-directed gene therapy as a treatment for infantile neuronal ceroid lipofuscinosis. J Inherit Metab Dis. 2012;35:847-57.

35. Levin SW, Baker EH, Zein WM, Zhang Z, Quezado ZM, Miao N, et al. Oral cysteamine bitartrate and $N$-acetylcysteine for patients with infantile neuronal ceroid lipofuscinosis: a pilot study. Lancet Neurol. 2014;13:777-87.

36. Sarkar C, Chandra G, Peng S, Zhang Z, Liu A, Mukherjee AB. Neuroprotection and lifespan extension in Ppt1(-/-) mice by NtBuHA: therapeutic implications for INCL. Nat Neurosci. 2013;16:1608-17.

37. Aldrich A, Bosch ME, Fallet R, Odvody J, Burkovetskaya M, Rama Rao KV, et al. Efficacy of phosphodiesterase-4 inhibitors in juvenile Batten disease (CLN3). Ann Neurol. 2016;80:909-23.

38. Kovacs AD, Weimer JM, Pearce DA. Selectively increased sensitivity of cerebellar granule cells to AMPA receptor-mediated excitotoxicity in a mouse model of Batten disease. Neurobiol Dis. 2006;22:575-85.

39. Kovacs AD, Saje A, Wong A, Szenasi G, Kiricsi P, Szabo E, et al. Temporary inhibition of AMPA receptors induces a prolonged improvement of motor performance in a mouse model of juvenile Batten disease. Neuropharmacology. 2011;60:405-9.

40. Kovacs AD, Saje A, Wong A, Ramji S, Cooper JD, Pearce DA. Age-dependent therapeutic effect of memantine in a mouse model of juvenile Batten disease. Neuropharmacology. 2012;63:769-75.

41. Tamaki S, Jacobs Y, Dohse M, Capela A, Cooper J, Reitsma M, et al. Neuroprotection of host cells by human central nervous system stem cells in a mouse model of infantile neuronal ceroid lipofuscinosis. Cell Stem Cell. 2009;5:310-9.

42. Selden NR, Al-Uzri A, Huhn SL, Koch TK, Sikora DM, NguyenDriver MD, et al. Central nervous system stem cell transplantation for children with neuronal ceroid lipofuscinosis. J Neurosurg Pediatr. 2013;11:643-52.

43. Griffey M, Bible E, Vogler C, Levy B, Gupta P, Cooper J, et al. Adeno-associated virus 2-mediated gene therapy decreases autofluorescent storage material and increases brain mass in a murine model of infantile neuronal ceroid lipofuscinosis. Neurobiol Dis. 2004;16:360-9.

44. Macauley SL, Roberts MS, Wong AM, McSloy F, Reddy AS, Cooper JD, et al. Synergistic effects of central nervous systemdirected gene therapy and bone marrow transplantation in the murine model of infantile neuronal ceroid lipofuscinosis. Ann Neurol. 2012;71:797-804.

45. Shyng C, Nelvagal HR, Dearborn JT, Tyynela J, Schmidt RE, Sands MS, et al. Synergistic effects of treating the spinal cord and brain in CLN1 disease. Proc Natl Acad Sci USA. 2017;114:E5920-9.

46. Katz ML, Tecedor L, Chen Y, Williamson BG, Lysenko E, Wininger FA, et al. AAV gene transfer delays disease onset in a TPP1-deficient canine model of the late infantile form of Batten disease. Sci Transl Med. 2015;7:313ra180.

47. Katz ML, Johnson GC, Leach SB, Williamson BG, Coates JR, Whiting REH, et al. Extraneuronal pathology in a canine model of CLN2 neuronal ceroid lipofuscinosis after intracerebroventricular gene therapy that delays neurological disease progression. Gene Ther. $2017 ; 24: 215-23$
48. Sondhi D, Johnson L, Purpura K, Monette S, Souweidane MM, Kaplitt MG, et al. Long-term expression and safety of administration of AAVrh.10hCLN2 to the brain of rats and nonhuman primates for the treatment of late infantile neuronal ceroid lipofuscinosis. Hum Gene Ther Methods. 2012;23:324-35.

49. Bosch ME, Aldrich A, Fallet R, Odvody J, Burkovetskaya M, Schuberth $\mathrm{K}$, et al. Self-complementary AAV9 gene delivery partially corrects pathology associated with juvenile neuronal ceroid lipofuscinosis (CLN3). J Neurosci. 2016;36:9669-82.

50. Sondhi D, Scott EC, Chen A, Hackett NR, Wong AM, Kubiak A, et al. Partial correction of the CNS lysosomal storage defect in a mouse model of juvenile neuronal ceroid lipofuscinosis by neonatal CNS administration of an adeno-associated virus serotype rh.10 vector expressing the human CLN3 gene. Hum Gene Ther. 2014;25:223-39.

51. Mitchell NL, Russell KN, Wellby MP, Wicky HE, Schoderboeck $\mathrm{L}$, Barrell GK, et al. Longitudinal in vivo monitoring of the CNS demonstrates the efficacy of gene therapy in a sheep model of CLN5 Batten disease. Mol Ther. 2018;26:2366-78.

52. Shevtsova Z, Garrido M, Weishaupt J, Saftig P, Bahr M, Luhder F, et al. CNS-expressed cathepsin D prevents lymphopenia in a murine model of congenital neuronal ceroid lipofuscinosis. Am J Pathol. 2010;177:271-9.

53. Arrant AE, Onyilo VC, Unger DE, Roberson ED. Progranulin gene therapy improves lysosomal dysfunction and microglial pathology associated with frontotemporal dementia and neuronal ceroid lipofuscinosis. J Neurosci. 2018;38:2341-58.

54. Worgall S, Sondhi D, Hackett NR, Kosofsky B, Kekatpure MV, Neyzi N, et al. Treatment of late infantile neuronal ceroid lipofuscinosis by CNS administration of a serotype 2 adeno-associated virus expressing CLN2 cDNA. Hum Gene Ther. 2008;19:463-74.

55. Bronson RT, Donahue LR, Johnson KR, Tanner A, Lane PW, Faust JR. Neuronal ceroid lipofuscinosis (nclf), a new disorder of the mouse linked to chromosome 9. Am J Med Genet. 1998;77:289-97.

56. Kleine Holthaus SM, Ribeiro J, Abelleira-Hervas L, Pearson RA, Duran Y, Georgiadis A, et al. Prevention of photoreceptor cell loss in a Cln6(nclf) mouse model of Batten disease requires CLN6 gene transfer to bipolar cells. Mol Ther. 2018;26:1343-53.

57. Wiley LA, Burnight ER, Drack AV, Banach BB, Ochoa D, Cranston $\mathrm{CM}$, et al. Using patient-specific induced pluripotent stem cells and wild-type mice to develop a gene augmentation-based strategy to treat CLN3-associated retinal degeneration. Hum Gene Ther. 2016;27:835-46.

58. Ranta S, Zhang Y, Ross B, Lonka L, Takkunen E, Messer A, et al. The neuronal ceroid lipofuscinoses in human EPMR and mnd mutant mice are associated with mutations in CLN8. Nat Genet. 1999;23:233-6.

59. Meyer JS, Katz ML, Maruniak JA, Kirk MD. Embryonic stem cell-derived neural progenitors incorporate into degenerating retina and enhance survival of host photoreceptors. Stem Cells. 2006;24:274-83.

60. Jankowiak W, Kruszewski K, Flachsbarth K, Skevas C, Richard G, Ruther K, et al. Sustained neural stem cell-based intraocular delivery of CNTF attenuates photoreceptor loss in the nclf mouse model of neuronal ceroid lipofuscinosis. PLoS One. 2015;10:e0127204.

61. Wen R, Tao W, Li Y, Sieving PA. CNTF and retina. Prog Retin Eye Res. 2012;31:136-51.

62. Groh J, Kuhl TG, Ip CW, Nelvagal HR, Sri S, Duckett S, et al. Immune cells perturb axons and impair neuronal survival in a mouse model of infantile neuronal ceroid lipofuscinosis. Brain. 2013;136:1083-101.

63. Groh J, Ribechini E, Stadler D, Schilling T, Lutz MB, Martini R. Sialoadhesin promotes neuroinflammation-related disease progression in two mouse models of CLN disease. Glia. 2016;64:792-809. 
64. Mirza M, Volz C, Karlstetter M, Langiu M, Somogyi A, Ruonala $\mathrm{MO}$, et al. Progressive retinal degeneration and glial activation in the CLN6 (nclf) mouse model of neuronal ceroid lipofuscinosis: a beneficial effect of DHA and curcumin supplementation. PLoS One. 2013;8:e75963.

65. Tracy CJ, Whiting RE, Pearce JW, Williamson BG, Vansteenkiste DP, Gillespie LE, et al. Intravitreal implantation of TPP1-transduced stem cells delays retinal degeneration in canine CLN2 neuronal ceroid lipofuscinosis. Exp Eye Res. 2016;152:77-87.

66. Whiting RE, Narfstrom K, Yao G, Pearce JW, Coates JR, Castaner $\mathrm{LJ}$, et al. Enzyme replacement therapy delays pupillary light reflex deficits in a canine model of late infantile neuronal ceroid lipofuscinosis. Exp Eye Res. 2014;125:164-72.
67. Griffey M, Macauley SL, Ogilvie JM, Sands MS. AAV2-mediated ocular gene therapy for infantile neuronal ceroid lipofuscinosis. Mol Ther. 2005;12:413-21.

68. Kohlschütter A, Schulz A, Denecke J. Epilepsy in neuronal ceroid lipofuscinoses (NCL). J Pediatr Epilepsy. 2014;3:199-206.

69. Williams RE, Adams HR, Blohm M, Cohen-Pfeffer JL, de Los Reyes E, Denecke J, et al. Management strategies for CLN2 disease. Pediatr Neurol. 2017;69:102-12.

70. von Tetzchner S, Elmerskog B, Tøssebro A, Rokne S, editors. Juvenile neuronal ceroid lipofuscinosis, childhood dementia and education. Snøfugl forlag: Melhus; 2019. 